\title{
Proust, Ponge, Jaccottet: un parcours phénoménologique dans le Visible
}

\section{Nathalie Aubert}

\section{(2) OpenEdition}

1 Journals

\section{Édition électronique}

URL : https://journals.openedition.org/studifrancesi/40176

DOI : $10.4000 /$ studifrancesi. 40176

ISSN : 2421-5856

Éditeur

Rosenberg \& Sellier

\section{Édition imprimée}

Date de publication : 1 juillet 2004

Pagination : 118-127

ISSN : 0039-2944

\section{Référence électronique}

Nathalie Aubert, « Proust, Ponge, Jaccottet: un parcours phénoménologique dans le Visible », Studi Francesi [En ligne], 142 (XLVIII | I) | 2004, mis en ligne le 30 novembre 2015, consulté le 07 décembre 2022. URL : http://journals.openedition.org/studifrancesi/40176 ; DOI : https://doi.org/10.4000/ studifrancesi.40176

\section{(c) (i) $\odot$}

Creative Commons - Attribution - Pas d'Utilisation Commerciale - Pas de Modification 4.0 International - CC BY-NC-ND 4.0

https://creativecommons.org/licenses/by-nc-nd/4.0/ 


\section{Proust, Ponge, Jaccottet: un parcours phénoménologique dans le Visible}

Si on considère la poésie de Rimbaud comme une dénonciation de l'unité ontologique du réel en ce que l'être réel est mis en rapport avec le principe de non-identité1 alors, on peut sans doute dire de la poésie moderne qu'elle est une radicalisation de la prise de conscience rimbaldienne. Le poète ne parle plus au nom d'un ensemble, il ne parle même plus en vue de cet ensemble mais pour mettre à jour une réalité qu'il est seul à pouvoir atteindre, dont seul il détient la clef.

Cinquante ans plus tard, dans le contexte différent d'une réflexion sur la création romanesque, Proust a laissé une page qui situe parfaitement la singularité du regard créateur:

Quant au livre intérieur des signes inconnus [...] pour la lecture desquels personne ne pouvait m'aider d'aucune règle, cette lecture consistait en un acte de création où nul ne peut nous suppléer ni même collaborer avec nous. Aussi combien se détournent de l'écrire! Que de tâches n'assume-t-on pas pour éviter celle-là! Chaque événement, que ce fût l'affaire Dreyfus, que ce fût la guerre, avait fourni d'autres excuses aux écrivains pour ne pas déchiffrer ce livrelà, ils voulaient assurer le triomphe du droit, refaire l'unité morale de la nation, n'avaient pas le temps de penser à la littérature. Mais ce n'étaient que des excuses, parce qu'ils n'avaient pas, ou plus, de génie, c'est-à-dire d'instinct. Car l'instinct dicte le devoir et l'intelligence fournit les prétextes pour l'éluder. Seulement les excuses ne figurent point dans l'art, les intentions n'y sont pas comptées, à tout moment l'artiste doit écouter son instinct, ce qui fait que l'art est ce qu'il y a de plus réel, la plus austère école de la vie, et le vrai Jugement dernier. Ce livre, le plus pénible de tous à déchiffrer, est aussi le seul que nous ait dicté la réalité, le seul dont «l'impression» ait été faite en nous par la réalité même².

Le narrateur privilégie comme seule vérité le rapport personnalisé du je à la réalité. Selon lui, la seule vraie réalité est celle qui procède du point de vue non substituable d'une conscience et d'une mémoire privées, particulières. Sa tâche consistera en une approche de l'irrécusable authenticité du monde et sera vécue comme quête de la Vérité. Le point de départ de la recherche n'est pas, comme chez Platon, une comparaison qui permettra aussitôt d'éliminer les caractères individuels pour ne plus garder que les caractères communs, mais une pénétration aussi complète que possible de l'individuel. Son corollaire est une réflexion sur l'effondrement ontologique du réel, qui mène Proust, mais aussi, et c'est ce que nous aimerions montrer ici, deux poètes à la fois différents et proches Francis Ponge et Philippe Jaccottet, héritiers de la démarche de l'auteur d' A la recherche du temps perdu qui font de l'individualité, et de son creusement toujours plus actualisé, particularisé, le centre de leur poésie.

\section{Le contact avec le monde}

Le rapport au réel est pensé par Proust, Ponge et Jaccottet en termes de contact, et de contact d'abord énigmatique, car il n'y a pas de possession immédiate de sa

(1) C'est entre autres explications possibles, un des sens du célèbre «Je est un autre» de la lettre à Georges Izambard, 13 mai 1871. Arthur Rimbaud, «Choix de lettres», Euvres, Paris, Garnier, 1960, p. 343-344.
(2) Marcel Proust, $A$ la recherche du temps perdu, édition établie sous la direction de Jean-Yves Tadié, Paris, Gallimard, 1987-1989 («Bibliothèque de la Pléiade»), 4 vol; ici t. IV, p. 458. 
substance. Parce qu'il est d'abord énigmatique, il est initiateur d'un retournement sur les moyens mis en oeuvre pour en rendre compte. Parce qu'il est d'abord énigme ou choc initial, il est questionnement sur le langage dans sa capacité de restitution de l'impression première. La poésie est donc pour Jaccottet et Ponge, comme l'écriture pour Proust, un travail de restitution et non une simple effusion subjective. En ce sens, on est justifié à évoquer l'auteur d'A la recherche du temps perdu dans cette démarche, bien qu'il ne soit pas, à proprement parler, poète, mais là encore, Ponge lui-même refuse catégoriquement cette appellation:

Le jour où l'on voudra bien admettre comme sincère et vraie la déclaration que je fais à tout bout de champ que je ne me veux pas poète, que j'utilise le magma poétique, mais pour m'en débarrasser, que je tends plutôt à la conviction qu'aux charmes, qu'il s'agit pour moi d'aboutir à des formules claires et impersonnelles, on me fera plaisir, on s'épargnera bien des discussions oiseuses à mon sujet ${ }^{3}$.

Tous trois sont sollicités par l'appel du monde sensible, ce qu'on pourrait appeler pour emprunter à Joyce un très beau terme, par les épiphanies du monde visible. Or, que sont-elles? Elles proposent à qui en reçoit la révélation, certains aspects concrets de la réalité:

Je fus saisi, plus violemment et plus continûment surtout qu'autrefois, par le monde extérieur. Je ne pouvais plus détacher mes yeux de cette demeure mouvante, changeante, et je trouvais dans sa considération une joie et une stupeur croissantes; je ne puis vraiment parler de splendeur, bien qu'il se soit toujours agi de paysages très simples, dépourvus de pittoresque, de lieux plutôt pauvres et d'espaces mesurés ${ }^{4}$.

Mais ces aspects sont en soi des plus fugitifs et se bornent à présenter leur évidence et cependant, même s'ils sont fragmentaires, ils parlent, pour le temps assez court de leur durée «au nom du tout».

Or, cette splendeur m'apparaissait de plus en plus lumineuse, aérée, et en même temps de moins en moins compréhensible. De nouveau, ce mystère nourricier, ce mystère réjouissant me poussait comme d'une poigne très vigoureuse vers la poésie; plus il semblait se dérober à l'expression, plus je ressentais le besoin de l'exprimer quand même, comme si le travail que j'aurais à faire sur les mots pour y parvenir allait m'aider à l'approcher, c'est-à-dire, aussi bien, à être de plus en plus réel.

Les épiphanies sont intuitivement pressenties comme siège de l'authentique beauté, manière très dialectique donc, de glorifier le monde visible dans le même temps où est affirmée sa bassesse, l'humilité de son statut ontologique. Francis Ponge souligne que «parler le regard» ou, plus précisément encore, et selon l'expression qu'il a formée lui-même, «le regard-de-telle-sorte-qu'on le-parle» ${ }^{6}$ est une réponse à «la muette supplication, les muettes instances que (les choses) font qu'on les parle, à leur valeur, et pour elles-mêmes, - en dehors de leur valeur habituelle de signification, - sans choix mais pourtant avec mesure, mais quelle mesure: la leur propre» (ibid).

Le contact avec les choses n'est donc pas au début de la langue mais «au bout de son effort, et en ce sens l'existence d'une langue donnée nous masque plutôt qu'elle ne nous montre la vraie fonction de la parole» ${ }^{7}$. Et cette tâche est douloureuse: il y a

(3) Francis PONGE, «My creative method», Le grand recueil, Paris, Gallimard, 1961, p40-41.

(4) Philippe Jaccottet, La promenade sous les arbres, Lausanne, Mermod, 1961, pp. 18-19 ensuite PSA.
(5) Ibid.

(6) Francis Ponge, «Les façons du regard», Proêmes, écrit en 1927, recueil publié en 1948 par Gallimard, Paris, p33-34.

(7) Maurice Merleau-Ponty, La prose $d u$ 
toujours, dans la perception, un moment d'adéquation, puis l'incapacité, momentanée peut-être, mais réelle, à dire l'épiphanie dans sa présence sans faille, qui consacre la perte irrémédiable et la ruine du sujet. C'est ce dont le narrateur a fait maintes fois l'expérience; ainsi, au début de A l'ombre des jeunes filles en fleurs:

Et comme d'autre part nous voulons continuer à penser à elle (une impression agréable), il (notre esprit) préfère l'imaginer dans l'avenir, préparer habilement les circonstances qui pourront la faire renaitre, ce qui ne nous apprend rien sur son essence, mais nous évite la fatigue de la recréer en nous- mêmes et nous permet d'espérer la recevoir de nouveau du dehors ${ }^{8}$.

De même, Jaccottet a connu ces moments de rencontre dans le visible qui sont comme un mouvement hors de soi/en soi, mais mouvement sans déplacement, pourvu d'une durée précaire, éloignée de toute position d'équilibre:

Un oiseau se mit à chanter; ce chant semblait une parole, et j'essayais de l'imiter en sifflant, sachant bien qu'il faudrait ne pas l'oublier et essayer, plus tard, d'en comprendre l'éventuelle signification".

Il ne s'agit pas de s'exprimer soi-même, à partir de ce qu'on est, de ce qu'on croit être, il faut sortir de soi et rendre compte de ce contact avec le monde.

Ponge, se montre conscient lui aussi du risque de perte si ce que la vision a enregistré n'est pas traduit par des mots:

J'ai d'abord eu, une fois - et je pourrais situer cela exactement dans le temps et dans l'espace -, une émotion me venant d'un pré, au sens de prairie. J'ai commencé à vouloir en rendre compte, éterniser si vous voulez, en quelque façon, par un texte, cette émotion, la garder devant moi et pour moi, écrire cela de peur de ne pas le retrouver. J'ai donc commencé à écrire, sans me préoccuper d'autre chose que de rendre compte de cette émotion. C'était une besogne d'expression, comme un paysagiste, mais évidemment en me servant des mots ${ }^{10}$.

\section{D'un lien organique avec le monde}

Proust, Ponge et Jaccottet indiquent tous trois que la véritable expression nécessite une opération de conscience, mais définie comme prise d'emblée dans l'opération perceptive, inhérente au jeu corporel. Cependant, le paradoxe de la présence est qu'elle ne peut se dévoiler que dans le retrait de cette opération de distanciation et d'emprise et que, au moment où le poète/l'écrivain veut parler de cette présence pure et sans distance, il rend compte de ceci précisément qu'il en parle, et donc ne se confond plus avec elle. Cette conscience qui «habite» le monde (jamais pure, toujours prise dans la facticité) mais qui ne le transforme pas, n'est cependant pas vide ou neutre: c'est, profondément, une conscience désirante. Dans le même temps, «désirer, c'est rendre apparent un intervalle» ${ }^{11}$, c'est-à-dire qu'il y a, dans ce refus de la tentation kantienne et bergsonienne de la fusion (où le sujet méditant se fond avec l'objet sur lequel il médite ${ }^{12}$, une conception neuve de la subjectivité et un lien au lan-

monde, Paris, Gallimard, 1969, p. 155.

(8) Recherche, t. II, pp. 18-19.

(9) Philippe Jaccottet, «Observations et autres notes anciennes», Observations III, Paris, Gallimard, 1998, p. 96.
(10) Entretiens Francis Ponge Philippe Sollers, Paris, Gallimard-Seuil, 1970, p. 172 (ensuite P/S).

(11) Georges Poulet, L'espace proustien, Paris, Gallimard, 1963, p. 61.

(12) Ce qui la sépare de tout idéalisme tradition- 
gage conçu en rapport à cette inhérence saturée de désir. Ponge le reconnaît aisément lorsqu'interrogé par Sollers il explique que ce qui l'amène «à franchir le silence», c'est «évidemment le désir» qu'il définit comme «quelque chose de quasi physiologique, biologique» et qui doit mourir pour que «le texte puisse naître» ${ }^{13}$. Déjà chez Proust, et ce fut sans doute l'un des traits fondateurs de sa modernité, le héros dont l'ambition est de devenir écrivain est dans une certaine mesure, aliéné à la transcendance propre du langage (de la structure de la langue) mais en même temps, le «sens» que ce dernier exprime n'est pas autre chose que la substance ontologique du monde en tant qu'elle existe à travers son corps. C'est pourquoi, l'auteur d'A la recherche du temps perdu, voit dans le miracle de la «transsubstantiation des qualités irrationnelles de la matière et de la vie dans des mots humains» ${ }^{14}$ une sorte de réussite stylistique absolue. Il y a un sens autochtone du monde qui se constitue dans le rapport avec lui de notre existence incarnée, et ce lien organique est le fondement, l'ancrage, de toute parole langagière visant à le dire. Cette conception que les trois auteurs partagent, phénoménologique dans son inspiration et ses buts, rompt avec la coïncidence intuitive dont parlait Bergson (s'inspirant d'ailleurs de Rousseau) et indique la direction d'un sens toujours inachevé, qui se manifeste sur un fond de non-sens toujours possible (précisément, comme je le disais tout à l'heure, si on ne parvient pas à l'expression).

Chez Ponge, la mutité du monde est d'abord l'indice d'une énigme pour celui qui parle, et non l'altérité d'un monde objectif privé de sens mais susceptible d'être entièrement éclairé par la raison (ce en quoi consiste le matérialisme, et je dis cela au passage parce qu'on a un peu trop eu tendance à faire de Ponge un matérialiste: il y a matérialisme, dans une certaine mesure chez Ponge car il pose un factualité qui résiste au sens, ce qui ne veut pas dire qu'elle soit entièrement privée de sens). La matière pour Ponge demeure un point aveugle, une limite. Il déploie donc en ce sens un questionnement de la limite, de son énigme, à ceci près qu'avec lui, ce n'est pas l'Idée «au-delà» du phénomène qui est visée, mais l'opacité d'un tourbillon primordial de l'être qui est précisément le fait d'exister. Habiter la matière, c'est donc pour Ponge se porter à la limite où s'obscurcit le sens, en-deçà du système construit des significations, vers le moment apertural, unique, du monde et du langage, où s'origine tout principe de correspondance entre les choses et les mots: (et Jaccottet, évoque, à propos de Nioque de l'Avant Printemps, «ce Ponge incomparable du choc initial» ${ }^{15}$ :

Il ne s'agit pas de «rendre», de «représenter» le monde physique, si vous voulez, mais de présenter dans le monde verbal quelque chose d'homologue ${ }^{16}$.

Proust quant à lui, inscrit dès le début d'A la recherche du temps perdu sa foi dans le sensible comme puissance anonyme, antérieure à toute pensée, déjà de connivence avec le monde. En effet, lorsqu'il cherche à actualiser la conscience vécue à l'intérieur de l'expérience sensible qui veut se saisir de sa propre naissance, c'est-à-dire lorsqu'il se rend compte de la relation singulière du corps avec la totalité spatio-temporelle, il se découvre comme pris par elle et en elle ${ }^{17}$. Aussi indique-t-il d'emblée les dangers d'une intelligence qui croit pouvoir se libérer de son enracinement en substituant au sens équivoque de sa vie une signification idéelle et peut-être mensongère. L'intelligence, en pensant le monde, cesse par là même de l'habiter, de le hanter, et toute la

nel, c'est que l'opération de conscience est justement une opération, et que pour cette raison, elle n'est jamais entièrement donnée à elle-même.

(13) $P / S$, op. cit., p. 170-171.

(14) Lettre à Lucien Daudet, Correspondance générale, éditée par Philip Kolb, Paris, Plon, t.XII, 1913 , p. 342-343.
(15) Cahiers de l'Herne, Paris, 1986, p. 36.

(16) $P / S$, op. cit., p. 48.

(17) Voir par exemple le début d'A la Recherche du temps perdu: «Un homme qui dort, tient en cercle autour de lui le fil des heures, l'ordre des années et des mondes. Il les consulte d'instinct...» t. I, p 5 et suivantes. 
difficulté de l'entreprise réside alors dans le fait que si le monde (le visible) est de l'ordre de la présence, de la chair, l'écriture et en particulier l'image, elle, est de l'ordre de l'élaboration. C'est le sens de l'injonction jaccottienne: «Méfie-toi des images. Méfie-toi des fleurs. Légères comme les paroles. Peut-on jamais savoir si elles mentent, égarent, ou si elles guident?» ${ }^{18}$

C'est en même temps, pour tous les trois, «comme si l'accès au monde n'était que l'autre face d'un retrait» ${ }^{19}$. Car la mutité du monde est d'abord l'indice d'une énigme et non le signe de l'altérité d'un monde objectif privé de sens mais susceptible d'être entièrement éclairé par la raison. Certains de ces «objets herméneutiques» ${ }^{20}$ rappellent un souvenir enfoui (comme les arbres d'Hudimesnil par exemple), les autres invitent à découvrir un sens caché derrière l'apparence. Parés d'une «densité énigmatique», il se présentent sous le signe de l'ambivalence: ils provoquent l'exaltation et en même temps apparaissent comme incomplets au narrateur le laissant insatisfait s 'il n'a pas réussi à analyser le plaisir qu'ils déclenchent en lui:

Mais j'avais beau rester devant les aubépines à respirer, à porter devant ma pensée qui ne savait ce qu'elle devait en faire, à perdre, à retrouver leur invisible et fixe odeur, à m'unir au rythme qui jetait leurs fleurs, ici et là, avec une allégresse juvénile et à des intervalles inattendus comme certains intervalles musicaux, elles m'offraient indéfiniment le même charme avec une profusion inépuisable, mais sans me le laisser approfondir davantage, comme ces mélodies qu'on rejoue cent fois de suite sans descendre plus avant dans leur secret ${ }^{21}$.

En même temps, comme le dit Jaccottet, ces sollicitations mystérieusement venues du monde réel, bien qu'elles soient toujours perçues comme énigmatiques justifient, en quelque sorte la tâche du poète parce qu'elles légitiment l'existence d'un sens, qui simplement, reste à déchiffrer:

Je ne décris pas le monde visible comme j'ai prétendu quelquefois vouloir le faire, [...] seulement ce que saisit l'oeil au passage. Je m'entête plutôt, ou je suis entraîné [...] à saisir de fuyants, d'illusoires rapports, et tout ce qui a l'air dans ce monde de vouloir me faire signe, qui en tout cas me touche, bien avant réflexion; c'est ainsi - et je n'y puis rien, plutôt m'en réjouirais-je - que m'éclaire l'obscurité du monde, et que me portent ses énigmes ${ }^{22}$.

La poésie de Ponge procède du même type de préoccupation:

Il est $[\ldots]$ un de ces points de vue dans la perspective [...] où git peut-être sinon le modèle, du mons la méthode du nouveau genre (d'écriture). [...] Nous l'avons baptisé l'objeu. C'est celui où l'objet de notre émotion, placé d'abord en abîme, l'épaisseur vertigineuse et l'absurdité du langage, considérées seules, sont manipulées de telle façon que, par la multiplication intérieure des rapports, les liaisons formées au niveau des racines et les significations bouclées à double tour, soit créé le fonctionnement qui, seul, peut rendre compte de la profondeur substantielle, de la variété et de la rigoureuse harmonie du monde ${ }^{23}$.

Tous trois dénoncent, et Proust met en scène, à travers le cheminement de son narrateur, l'illusion de la réalité comme tout indivis, comme opacité d'abord, puis transparence. «Aller derrière», ou «au-delà», c'est concevoir le réel comme une sorte de texte chiffré, et non dans son épaisseur. Lorsque le héros d' A la recherche du temps perdu prend enfin conscience, dans Le temps retrouvé, que «seule la perception plutôt

(18) Philippe Jaccottet, $A$ travers un verger, Montpellier, Fata Morgana, 1975, p. 17.

(19) Maurice Merleau-Ponty, Le visible et l'invisible, Paris, Gallimard, 1964, p. 23.

(20) Jean-Pierre Richard, Proust et le monde sensible, Paris, Seuil, 1974, p. 155 et suivantes.

(21) Recherche, t. I, p. 136.

(22) PhilipPe JaccotTet, Observations et autres notes anciennes, Paris, Gallimard, 1998, p. 124-125.

(23) $P / S$, op. cit., p. 141. 
grossière et erronée place tout dans l'objet quand tout au contraire est dans l'esprit» ${ }^{24}$ cesse enfin l'illusion d'un «pur langage», d'un «au-delà» des langues «naturelles» comme dans la tradition platonicienne. Le rêve d'un déchiffrement du monde, n'est que l'envers de la même illusion qui consiste à séparer réel et conscience. La volonté de fonder une esthétique sur une figure comme la métaphore, l'ambition de se porter hors des confins de l'intelligence, hors des limites de sa lumière, amène nécessairement Proust à rechercher la justesse du rapport entre les deux réalités rapprochées moins dans la contingence hasardeuse d'une contiguïté que dans une nécessité immanente. Cette conception du contact ontologique conduit Proust (mais aussi bien Jaccottet et Ponge comme on va le voir dans un instant) à dénoncer ${ }^{25}$, l'erreur qui consiste, pour les poètes, à rechercher «cet air plus pur» qu'ils «ont vainement essayé de faire régner dans le paradis et qui ne pourrait donner cette sensation profonde de renouvellement que s'il avait été respiré déjà, car les vrais paradis sont les paradis qu'on a perdus». Jaccottet rejette ainsi cette façon de s'absenter au monde:

Je vis que A.E. ne questionnait pas réellement le monde, mais volait vers un monde «supérieur», et que ce monde «supérieur» avait tous les défauts (à mes yeux) de la sur-nature: en particulier, d'être exsangue (et comment en serait-il autrement?) Pour moi, j'avais cru voir le secret dans la terre, les clefs dans l'herbe. Sans doute ce qui nous attend à l'issue ne peut-il être conçu: mais je me dis qu'il fallait avancer dans la direction de cet inconcevable (qui nous fascine comme tout abîme) à travers l'épaisseur du Visible, dans le monde de la contradiction, avec des moyens et des sentiments ambigus, en particulier un mélange d'amour et de détachement, d'acharnement et de négligence, d'ambition et d'ironie ${ }^{26}$.

\section{Leur «creative method»}

A quoi ces trois écrivains rêvent-ils d'arriver? A une parole non médiatisée, une écriture de la présence qui s'inscrirait dans un axe extrêmement ténu, extrêmement mouvant aussi, dans l'entre-deux de la présence et de l'absence. La force expansive de la réminiscence offre au narrateur proustien un modèle enviable de reconstitution du tout, mais qui ne dure pas:

Et comme dans ce jeu où les Japonais s'amusent à tremper dans un bol de porcelaine rempli d'eau, de petits morceaux de papier jusque-là indistincts qui, à peine plongés s'étirent, se contournent, se colorent, se différencient, deviennent des fleurs, des maisons, des personnages consistants et reconnaissables, de même maintenant toutes les fleurs de notre jardin et celles du parc de M.Swann, et les nymphéas de la Vivonne, et les bonnes gens du village et leurs petits logis et l'église et tout Combray et ses environs, tout cela qui prend forme et solidité, est sorti, ville et jardins, de ma tasse de thée ${ }^{27}$.

Dans la métaphore, il entrevoit le moyen de «convertir» ${ }^{28}$ la sensation en un «équivalent spirituel $\gg^{29}$ précisément pour la soustraire au risque de l'évanescence. Comme instrument de recherche, la métaphore se situe au niveau de la découverte et de l'expression d'un sens qui n'est jamais définitivement garanti, qui se manifeste sur le fond d'un non-dit (d'un non-sens) toujours possible. Dans La Recherche en effet, la métaphore apparaît comme un outil de prospection et Proust en use comme d'une «sonde» ${ }^{30}$ lancée parfois plusieurs fois dans la même direction en une reprise entêtée pour parvenir à la formulation adéquate.

\author{
(24) Recherche, t. IV, p. 493. \\ (25) Ibid. \\ (26) La promenade sous les arbres, p. 40. \\ (27) Recherche, t. II, p. 47.
}

(28) Recherche, t. IV, p. 457.

(29) Ibid.

(30) Recherche, t. IV, p. 458 
Ponge est sans doute celui qui rejette avec le plus de virulence l'illusionnisme de la conception du poème comme objet parfait. Les petites pièces du Parti pris des choses, brèves, closes sur elles-mêmes, vont progressivement lui apparaître comme des «bibelots», un peu trop des poèmes justement. La clôture de ces textes, souvent marquée dans le texte lui-même par la présence d'un trait tiré sous le poème, finit par sembler à leur auteur artificielle, intenable. Le texte pongien, parce qu'il rend compte d'un contact, circonstancié, individuel avec le monde sensible, est ainsi toujours incomplet, insatisfaisant, donc recommençable, reformulable. Ainsi, au lieu de donner des textes courts, brefs, et qui ont l'apparence de choses closes, données comme telles, il a ensuite souvent préféré donner la relation de son échec, c'est-à-dire toute une suite de brouillons datés, où apparaît la sensation, individuelle, circonscrite dans le temps et dans l'espace.

Chez Jaccottet, il y a, dans $A$ travers un verger par exemple, une sorte d'opération semblable, mais même, rien ne prouve en fait qu'elle se résolve vraiment jamais en «poème»; c'est la recherche qui est le travail lui-même. Dans les carnets que Jaccottet publie régulièrement depuis 1963 , on s'aperçoit que ce qui triomphe, c'est un art de la reprise qui correspond à un retour peut-être plus proche de ce que Proust ambitionnait de faire avec la métaphore conçue comme arche qui relierait cette absence qui se fait sentir comme présence, le monde. L'art de la reprise inscrit ainsi la temporalité au coeur de son écriture, établissant la jonction entre le moi d'aujourd'hui et le moi d'autrefois. A l'encontre peut-être de Ponge, qui recherchait plutôt la tension contradictoire qui définissait un objet, un instant, la démarche de Jaccottet et de Proust tient beaucoup dans l'approche, car l'impression première a brusquement confronté l'écrivain à l'impossible, à l'invisible. Si le corps, dans une sensation muette peut entrer en contact avec ce qui, toujours, se retire, il revient à l'écriture qui suppose à la fois un écart et un rapprochement, un rapprochement par l'écart, de retracer ce contact. L'écriture est donc ce retournement, le lieu de ce retournement.

Bergson, qu'on a tellement associé à Proust, faisait ${ }^{31}$ de la mémoire une puissance souple et vivante seule à même de produire du sens. Toute perception attentive, selon lui, est moins une analyse qu'une suite de synthèses qui se projettent sur le donné et se confirment ou s'infirment par lui. Il avait bien aperçu cette naissance du sens dans une rencontre, mais ne s'était apparemment pas interrogé sur ce qui permet au passé de faire sens alors même que, d'après lui, la perception n'en a jamais par elle-même. Chez Proust cependant, la perception a du sens, il ne s'agit en aucun cas de rejoindre une hypothétique «sensation pure». Toute la tâche de l'écrivain à venir dans $L a$ Recherche est de parvenir à extraire telle sensation (individuelle, circonscrite dans le temps et l'espace) de façon satisfaisante: la métaphore proustienne, dans sa complexité, cherche à rejoindre l'intensité de l'impression originelle dans sa dimension de surprise, d'émoi et de questionnement de l'expression. L'enthousiasme qui l'avait fait s'écrier «zut, zut, zut, zut» lors de l'épisode de la mare de Montjouvain avait mis à sa disposition un mot -inadéquat- là où il cherchait une sensation, ou, ce qui revient au même chez Proust, une expression adéquate.

\section{La différence}

«Ces mystérieuses différences d'où dérive la beauté» ${ }^{32}$, qui constituent l'enjeu de sa conception du style, proviennent du fait que la mémoire involontaire n'a pas le seul passé pour objet, qu'elle met en mouvement chaque figure du passé par une 
mise en présence: ce qui se traduit par l'intégration, dans la forme même, de cette «rumeur» des «distances traversées» dont parle le héros dans le fameux passage sur la madeleine lorsque l'absorption du petit gâteau imbibé de thé le fait remonter vers la source de la sensation initiale ${ }^{33}$. Elles représentent les étapes successives par lesquelles cette image (et la réalité qu'elle cherche à exprimer) est passée avant d'aboutir à une expression dense où se concentre tout ce que la mémoire du narrateur a, précisément, «amassé». Elle est par conséquent travaillée par l'écart, l'intervalle, dont «l 'augmentation ou la diminution» est, chez Proust, le siège de «la beauté» ${ }^{34}$. L'image rend compte chez lui, du traumatisme de la vision, en retranscrit l'étrangeté. La métaphore, dans ce qu'on pourrait appeler la dissemblance de sa forme, jamais achevée, toujours, d'une certaine façon, à venir, porte en elle la trace du contact («petit trait» ou «petit sillon») avec le réel et c'est peut-être tout ce qu'elle peut en restituer. Vestige donc, mais aussi trace, présence, elle secrète en elle-même une tension, ne fût-ce que celle de désirer l'inaccessible image. Il nous semble que chez Proust, précisément en raison de l'étrangeté que confère à $L a$ Recherche sa dimension, en même temps que d'oeuvre achevée (et comme telle, affectée d'une valeur artistique), de livre encore «à venir», il est impossible de tirer son esthétique vers l'idéalisme platonicien ${ }^{35}$ car il n'est pas à la recherche de l'essence des choses: bien au contraire, comme dans la démarche phénoménologique, «la marche à l'adéquation, dont les faits de dés-illusion témoignent, n'est pas le retour en soi d'une Pensée adéquate qui se serait inexplicablement perdue de vue [...] c'est la prépossession d'une totalité qui est là avant qu'on sache comment et pourquoi $\gg^{36}$. La définition que Merleau-Ponty donne ici de la perception est au coeur même des conclusions auxquelles Proust a abouti car son ambition était d'insérer dans son oeuvre la totalité de l'expérience, erreurs comprises: la forme se présente alors comme le passé d'un passage, sa trace. Une fois l'illusion démasquée, cette dernière est intégrée à la «nouvelle» réalité, celle aussi d'un langage qui, en ce sens, serait tâche autant qu'héritage. C'est dans la tresse de l'illusion et de la désillusion, dans l' incessant passage entre surface et profondeur rétablissant la dignité de l'apparence (c'est-à-dire dignité de la surface, de l'aspect reconnus comme modes de vestige) que la limite du texte proustien ne cesse de vaciller. Mieux valent les erreurs, l'inconnaissance assumées qu'un simulacre de la philosophie des essences car chez Proust, la métaphore est pensée aux fins de montrer l'altérité du réel, ce qui est tout autre chose que de croire en signifier l'essence. C'est par là, par cette frange de non-être qu'elle évoque l'approche phénoménologique. Chez l'auteur de La_Recherche, la métaphore a pour fonction d'intégrer en premier lieu, depuis la fragilité (l'incertitude) de la perception de ce personnage qui dit «je», le signe de l'apparence visible, celle qu'appréhende l'oeil charnel et, au-delà, le signe d'une cause (c'est le sens de la série des «révélations») qu'appréhende la raison. Le cheminement vers la vérité du style que le narrateur dit rechercher est cette opération permettant à la fois de préserver la transcendance du mystère de l'écriture et de faire jouer constamment l'immanence de la visualité des figures. Car, placer l'absolu du style dans la métaphore, c'est faire du visuel l'instrument par excellence de l'entrelacs où s'exacerbe le proche, dans le jeu de l'équivoque signifiante, pour représenter le lointain, l'indicible altérité d'un au-delà (d'un virtuel) qui est l'oeuvre ${ }^{37}$.

Comment ne pas voir, dès lors, dans cette conception du style une affinité profonde avec la démarche de Ponge et de Jaccottet? L'écriture est ce qui permet

(33) Recherche, t. I, p. 45.

(34) Ibid., p. 632.

(35) Il est vrai que toute une terminologie d'ailleurs datée, directement issue d'un puissant courant spiritualiste dont nous mesurons mal aujourd'hui quelle fut alors l'importance, peut par- fois s'y prêter dans le texte.

(36) Le visible et l'invisible, op. cit., p. 65.

(37) L'image est toujours aussi, en même temps, «en vue de l'image», image «à venir» et, en ce sens, elle est dotée d'une vertu «anagogique'. 
précisément de faire voir, de rendre compte de la complexité de notre relation au monde. Tous deux ne croient pas aux effets de l'analogie: par exemple, Ponge «la qualité différentielle [est] plus intéressante que la qualité analogique» ${ }^{38}$ ou bien «les différences, c'est plus intéressant que les analogies». Il explique que sa démarche se rapproche de l'idée d'un «dictionnaire phénoménologique»:

Car ce sont bien des définitions que je prétends formuler, mais telle que, n'impliquant nullement que j'aie fait d'abord table rase, mais plutôt rassemblé, au contraire, en un premier temps, les connaissances déjà élaborées, aussi bien en moi-même, sur chaque sujet, elles contiennent également des éléments nouveaux et, si l'on veut, une part du futur de nos connaissances sur le même sujet. Mais comment y parviens-je, si j'y parviens? (Voici le point important) En repétrissant avec les connaissances anciennes les acceptions morales et symboliques, et toutes les associations d'idées, la plupart du temps très variées et contradictoires, auxquelles cette notion peut ou a pu donner lieu, y compris celles habituellement considérées comme puériles, gratuites et sans intérêt, celles-là mêmes de préférence peut-être, parce que ayant plus de chance d'apporter quelque élément non encore utilisé. Si bien que, par l'agglomérat [on remarque que Ponge parle d' «agglomérat» là où Proust utilise le participe passé «amassé»] de toutes ces qualités ou qualifications contradictoires, et plus elles sont contradictoires et semblent irrationnelles, mieux cela vaut, j'obtiens un conglomérat neutre, dépourvu de toute tendance ou résonance morale propre à offusquer les vérités nouvelles et inouïes dont je désire passionnément qu'elles s'y incorporent, et, de la sorte, effectivement, elles s'y incorporent» ${ }^{39}$.

Par un long cheminement qui s'étend parfois sur des années, la réalité d'un objet, d'un moment, est re-captée. Une certaine inflation, celle des brouillons, des repentirs, vise à l'affirmation consistante de la «qualité différentielle» qui chez lui, est aussi bien le sentiment de la différence entre le mot et la chose: «Ce qui nous fait reconnaître une chose comme chose, c'est le sentiment qu'elle est différente de son nom, du mot qui la désigne, du mot qui porte son nom, du mot dont elle est bien touchante de consentir à porter le nom» ${ }^{40}$. Pour Ponge en effet, les «phénomènes du monde extérieur» sont déjà des mots: mouvement en soi/hors de soi qui est fondateur de l'approche phénoménologique et qu'il exprime ainsi: «Nous ne [...] nous extasions devant elles [les choses] que dans la mesure où nous les re-connaissons. Le mouvement [l'émotion] qui se fait en nous [qu'elles suscitent en nous] et qui nous les fait à la fois reconnaître comme semblables à leur nom et connaître (avec surprise) c.à.d. découvrir comme différentes de leur nom, qui nous fait, par conséquent, désirer les nommer mieux «se traduit», en fait, par une attention redoublée à leur nom, qui serait tout simplement à rendre sa signification première (ou complète), afin de le rapprocher à nouveau de la chose, conçue dans son épaisseur et sa différence véritables: celles qui la caractérisaient quand elle fut nommée pour la première fois ${ }^{41}$. Qu'est-ce, en ce sens, que la tâche que s'assigne le poète si ce n'est créer des oeuvres qui expriment une même vibration: elles sont évidemment liées au temps, elles retrouvent le temps perdu sans cacher que d'une certaine manière, il est perdu, sans dire non plus que d'une autre manière, il est retrouvé:

J'ai d'abord eu, une fois - et je pourrais situer cela exactement dans le temps et dans l'espace -, une émotion me venant d'un pré, [...] J'ai commencé à vouloir en rendre compte, éterniser si vous voulez, en quelque façon, par un texte, cette émotion, la garder devant et pour moi, écrire cela de peur de ne pas le retrouver. J'ai donc commencé à écrire, sans me préoccu-

(38) Francis Ponge, Nioque de l'avant printemps, Paris, Gallimard, 1983, mais texte de 1950.

(39) $P / S$, op. cit., p. 131-132.
(40) Francis Ponge, La fabrique du pré, Genève, Skira, 1970, p. 23-24.

(41) Ibid., p. 22. 
per d'autre chose que de rendre compte de cette émotion. C'était une besogne d'expression, comme un paysagiste, mais évidemment, en me servant des mots. Très vite, je me suis aperçu que ce mot, ce mot lui-même, pré, qui revenait dans mes notes, ce mot devenait par lui-même non seulement intéressant, mais enfin, devenait pour moi comme une espèce d'obsession. $\mathrm{Ce}$ mot> ${ }^{42}$.

C'est au lent travail de la remontée des mots qu'est confiée la charge de rendre visible ce pré. Et ce travail des mots, il ne peut être qu'un travail de reprise. Il me semble que c'est précisément la démarche de Jaccottet dans le texte intitulé L'approche des montagnes dans La promenade sous les arbres. Si le travail de l'expression cherche à rendre hommage à ces montagnes, c'est bien en essayant non pas de prendre la montagne comme le mot «montagne» usuel, mais en essayant de travailler ce mot; et c'est ainsi que ces rochers, tout cet univers minéral aux masses pesantes, se transforment en une buée, une vapeur:

Mais vient un moment où la base des montagnes disparaît dans la lumière, de sorte qu'on ne voit plus que leur cîme, comme dans certaines peintures chinoises; alors est irrésistible, quoi que j'en aie, l'idée d'un «château des dieux», non pas tant dans un esprit proprement religieux, et surtout pas mythologique, qu'avec le sentiment du léger, du haut, de l'invisible ${ }^{43}$.

Or, ce travail sur l'expression ne peut se justifier qu'à une condition, qui est celle de trouver, pour le poète, le ton juste. Cette voix, cet «air de la chanson» comme disait Proust, est à la fois retenue, réticente, une voix de parole et de silence mêlés. La reprise dès lors, n'est plus qu'une marque de précaution, de respect, à l'égard du monde. Voir, c'est alors, écouter en nous, dans notre langue, la trace qu'y ont déposée les choses. Où l'on retrouve Proust.

\section{Conclusion}

Il y a, chez ces trois écrivains que nous avons choisi de rassembler, l'idée que l'écriture est quand même un des seuls modes d'accès au réel. Tout se joue entre l'épiphanie et le moment présent. L'accès aux choses passe par le cheminement d'autrefois: c'est cela la proposition proustienne de l'écriture, et en particulier de la métaphore qui est le recouvrement unique de ces deux éléments. Cet entre-deux, on le retrouve dans les textes de Ponge qui à la fois visent à dire quelque chose d'un objet et dans le même moment, montrent l'auteur essayant de tirer au clair les conditions de l'élaboration. Il existe alors une tension entre énigme et sens, et c'est précisément à ce point que la réversibilité peut se faire, dans la résistance de la signification. La vraie éternité, c'est celle qui est conjointe au sensible et au vécu. Le texte serait alors comme une traversée de l'espace sensible: chez Proust, c'est le temps qui est donné comme liaison ultime de tous les lieux, comme réunion des «deux côtés»; chez Ponge, c'est la rêverie sur le nom, puisqu'elle le mène, à travers les étymologies, à son idée de dictionnaire sensible. L'écriture est ainsi édification d'une terre de mémoire.

NATHALIE AUBERT 